\title{
CT-Guided Interventions Using a Free-Hand, Optical Tracking System: Initial Clinical Experience
}

\author{
Tilman Schubert • Augustinus L. Jacob • \\ Michele Pansini - David Liu - Andreas Gutzeit • \\ Sebastian Kos
}

Received: 8 June 2012/ Accepted: 15 November 2012/Published online: 12 December 2012

(C) Springer Science+Business Media New York and the Cardiovascular and Interventional Radiological Society of Europe (CIRSE) 2012

\begin{abstract}
Purpose The present study was designed to evaluate the geometrical accuracy and clinical applicability of a new, free-hand, CT-guided, optical navigation system.

Methods Fifteen procedures in 14 consecutive patients were retrospectively analyzed. The navigation system was applied for interventional procedures on small target lesions, in cases with long needle paths, narrow access windows, or when an out-of-plane access was expected. Mean lesion volume was $27.9 \mathrm{ml}$, and mean distance to target measured was $107.5 \mathrm{~mm}$. Eleven of 15 needle trajectories were planned as out-of-plane approaches regarding the axial CT plane.

Results Ninety-one percent of the biopsies were diagnostic. All therapeutic interventions were technically successful. Targeting precision was high with a mean distance of the needle tip from planned target of $1.98 \mathrm{~mm}$. Mean intervention time was $1: 12 \mathrm{~h}$. A statistically significant correlation between angular needle deviation and intervention time $(p=0.007)$, respiratory movement of the target $(p=0.008)$, and body mass index $(p=0.02)$ was detected. None of the evaluated parameters correlated
\end{abstract}

T. Schubert $(\bowtie)$ - A. L. Jacob · M. Pansini · S. Kos Department of Radiology and Nuclear Medicine, University Hospital Basel, 4031 Basel, Switzerland e-mail: TSchubert@uhbs.ch

D. Liu

Department of Radiology, Vancouver General Hospital, University of British Columbia, Vancouver, BC, Canada

\section{A. Gutzeit}

Department of Radiology, Winterthur Cantonal Hospital, Winterthur, Switzerland significantly with the distance from the needle tip to the planned target.

Conclusions The application of a navigation system for complex CT-guided procedures provided safe and effective targeting within a reasonable intervention time in our series.

Keywords Computed tomography · Navigation - Image guidance $\cdot$ Biopsy $\cdot$ Image-guided therapy

\section{Introduction}

Computed tomography (CT)-guided percutaneous procedures are widely used for a variety of different purposes. In general, they can be classified according to diagnostic or therapeutic intentions. Due to the reliable depiction of the target and structures along the needle path, CT-guided interventions are safe and precise [1,2]. However, these minimally invasive procedures require a certain experience of the operator, because the needle is advanced without any real-time guidance, only reliant on the previously acquired CT image. Furthermore, some conditions limit the accessibility even for the experienced interventionalist. Challenging characteristics of CT-guided interventions are out-of-plane approaches, long, narrow access pathways, and respiratory mobile targets. Out-of-plane approaches from the axial CT plane can be compensated to a certain degree by tilting the CT gantry [3]. However, this technique is limited by the maximal angulation achieved by the gantry. In addition, not all helical multislice CT systems are capable of modifying the reconstruction algorithms used to create CT images without significant artefacts when the gantry is tilted [4]. A technique introduced to overcome the problem of maximum gantry tilt is the triangulation 
method; however, a comparably high number of needle readjustments and multiple CT controls were reported [5]. An alternative technique to perform out-of-plane approaches is CT fluoroscopy (CTF) [6]. In contrast to intermittent CT controls, CTF allows real-time guidance of the needle with high precision. However, CTF is associated with high radiation exposure to both patient and interventionalist [7]. A more recently introduced technology to counter the high demands of out-of-plane approaches or very small targets with narrow access pathways are CT-guided navigations systems. In contrast to conventional, cross-sectional, image-guided procedures, navigation systems provide interactive visualization of the needle in a preoperatively acquired 3D-dataset. The determination of the virtual 3D-dataset to the patient's body and the probe is performed via fiducial markers on the patient's body and the needle captured by the navigation system $[8,9]$. Most navigation systems use surface markers on both the patient's skin and the probe that are captured by a camera device during intervention $[8,9]$. These systems can reduce needle misplacement and repeated puncture attempts [9]. Navigation systems provide high targeting precision that is especially important in minimally invasive therapeutic interventions $[9,10]$. For this type of procedure, targeting precision is essential, because it accounts for the therapy outcome [11]. Furthermore, navigation systems help to achieve reproducible results less reliant on the interventionalist's experience than freehand punctures [9]. However, current optical navigation systems cannot compensate for internal organ movement (i.e., respiratory) or elastic organ deformation. These effects are not reliably depicted by surface markers [12]. For this reason, internal markers, such as marker needles and electromagnetically traceable vein catheters have been introduced [12-16]. Indeed, such invasive devices may cause complications and have been reported as a highly time-consuming step within the workflow $[13,14]$. In the present study, the geometrical accuracy and clinical practicability of a CT-guided navigation system applied for complex CT-guided punctures has been evaluated.

\section{Materials and Methods}

\section{Patients and Lesions}

Fifteen CT-guided procedures with the described system were performed in 14 consecutive patients ( 8 females, 6 males) between January and June 2011. Regarding the total amount of 173 CT-guided interventions performed in our department within this period, the percentage of navigated procedures was $11.5 \%$. The procedures were retrospectively analyzed. Three radiologists with 2,5 , and 20 years of experience in interventional radiology performed the navigated procedures. The patients' ages ranged from 45 to 82 years (mean 64), the mean body mass index (BMI) was 25.4 (range 17.3-35.9). Interventions performed comprised of 11 diagnostic (biopsies) and 4 therapeutic interventions (1 abscess drainage, 1 thoracic vertebroplasty, and 2 nephrostomies; Table 1). One biopsy was performed after a previous, nonnavigated biopsy resulted not diagnostic. Application criteria of the navigation system were an expected out-of-plane approach of more than 5 degrees or when the projected target diameter of the approach window or lesion projected orthogonally to the needle path was below $1 \mathrm{~cm}$. Conscious sedation (Midazolam/Dormicum and Alfentanil/Rapifen) was administered intravenously if regarded necessary by the interventionalist. Vital parameters (heart rate, blood pressure, peripheral oxygenation) were continuously monitored. For all procedures but the vertebroplasty, for which an 11-gauge needle was utilized, 17-gauge coaxial introducer needles were used. For procedures on respiratory moving organs, the breath-hold technique was explained to the patient and practiced immediately before intervention.

Lesion volume was approximately calculated using the formula for ellipsoid-shaped bodies: $4 / 3 \times \pi(a \times b \times c)$; $\mathrm{a}, \mathrm{b}$, and $\mathrm{c}$ were the radii of the main axes.

\section{Navigation}

The applied, stereotactic, CT-guided navigation system (ActiViews, Haifa, Israel) has been commercially available since 2010 and received U.S. Food and Drug Administration marketing approval in 2011. The navigation system provides planning of virtual trajectories in a previously acquired CT-dataset as well as planning of entry and target point in the data volume.

After the planning procedure, a single-use reference pad with CT-visible fiducial markers is attached to the patient's skin. A specifically collimated navigation CT scan is then performed (slice thickness $1 \mathrm{~mm}, 96 \times 0.6$ collimation, pitch 1.4), where the definite needle trajectory is to be planned. Mapping of the biopsy probe is achieved via optical tracking. For this purpose, a miniature, single-use video camera is attached to the end of the probe. During navigation, the mapping of the camera and the probe are determined relative to the fiducial markers on the reference pad on the patient's skin. The combination of the above mapping enables determination of the needle position relative to the actual target (Fig. 1). The actual position of the probe in the navigation dataset is depicted in two planes on the navigation screen (Fig. 2). The needle course is controlled through intermittent $\mathrm{CT}$ scans as during purely manual interventions. All interventions were performed in 


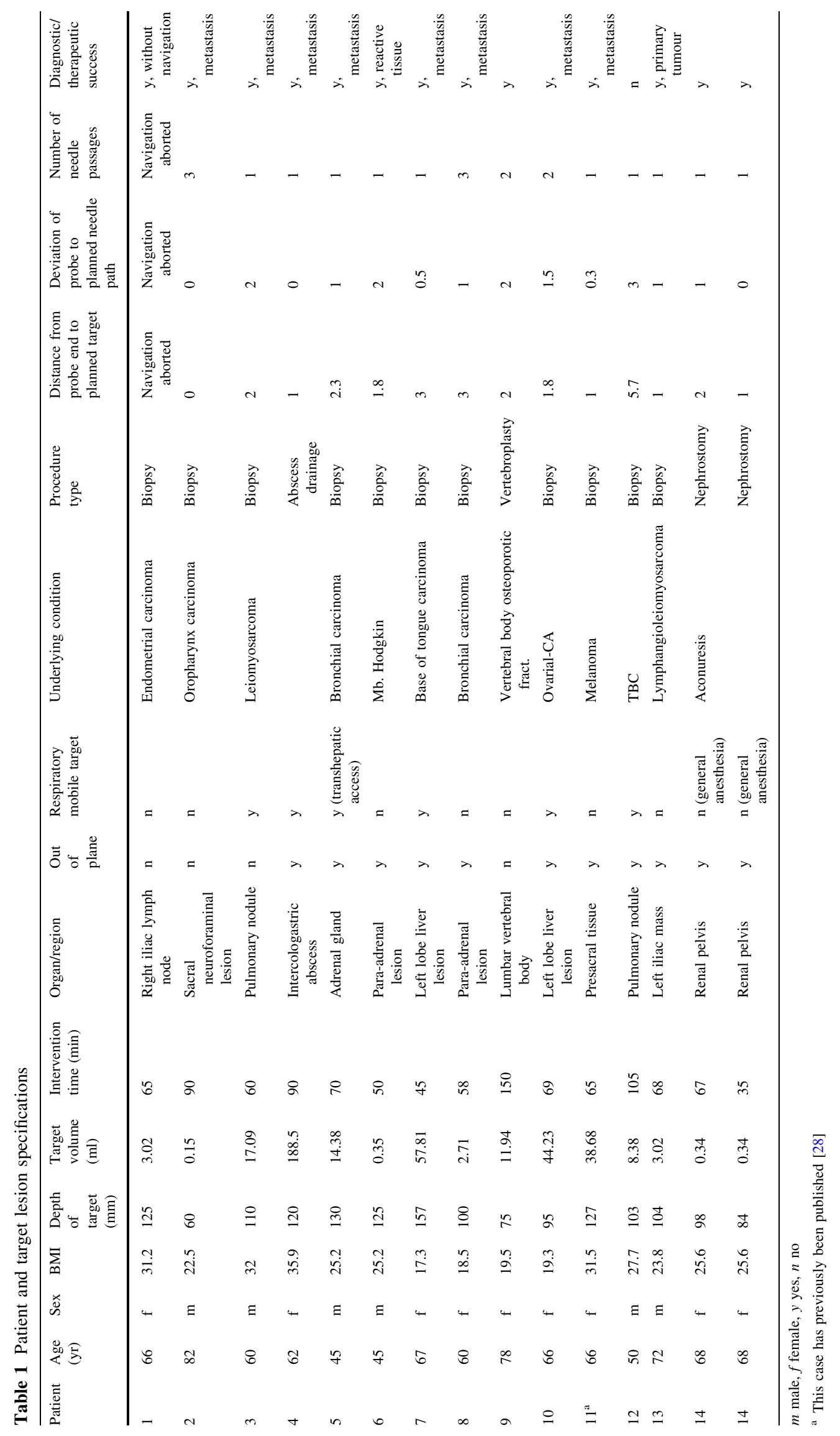




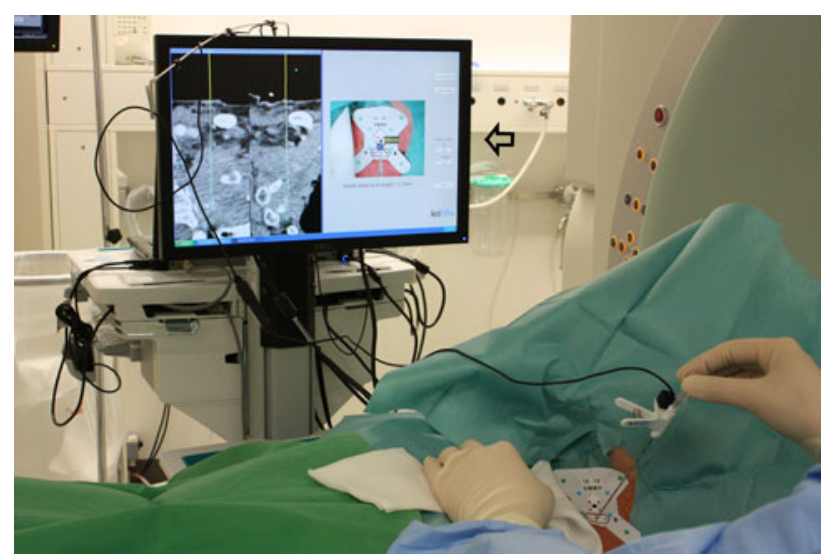

Fig. 1 Setup of the navigation system: the screen is located vis-a-vis to the interventionalist (empty arrow), usually on the contralateral side of the patient

a 64-slice CT scanner (Somatom Sensation 64, Siemens Medical Solutions, Erlangen, Germany).

Target and Planning Specifications

Mean lesion volume was $27.9 \mathrm{ml}$ (range 0.34-188.5). The mean distance from the skin to the target lesion measured $107.5 \mathrm{~mm}$ (range 75-157). Localization of the lesions is depicted in Table 1. Eleven of 15 trajectories were outof-plane approaches regarding the axial CT planes in contrast to 4 in-plane approaches.
Six targets were located in respiratory mobile body regions, and eight targets were located in body regions not influenced by respiratory motion (Table 1). One biopsy of a suprarenal gland was regarded as respiratory mobile because of the chosen transhepatic access. Two nephrostomies were regarded respiratory immobile because the (bilateral) procedure was performed under general anesthesia, and a reproducible breathing position could be achieved. A mean of 3.8 intermittent CT scans were performed during interventions.

\section{Measurement Methods}

Measurements of the deviation angle and the distance of the needle tip to the planned target were performed on the basis of procedural controls. After the intervention, the planned trajectory was manually plotted in the control scan with the needle in place by comparing the slice position of entry and target points for longitudinal position and anatomical landmarks and morphology of the target for axial position. If the needle was not advanced until or beyond the planned target, the closest distance from the needle trajectory to the target point was taken as distance from probe end to target. That distance was measured in a plane perpendicular to the planned trajectory (Fig. 3), which led to only one error value instead of lateral and longitudinal error values as depicted in other studies $[10,16]$. In case we counted more than one needle passage, we used the initial passage to measure the deviation angle of the final probe

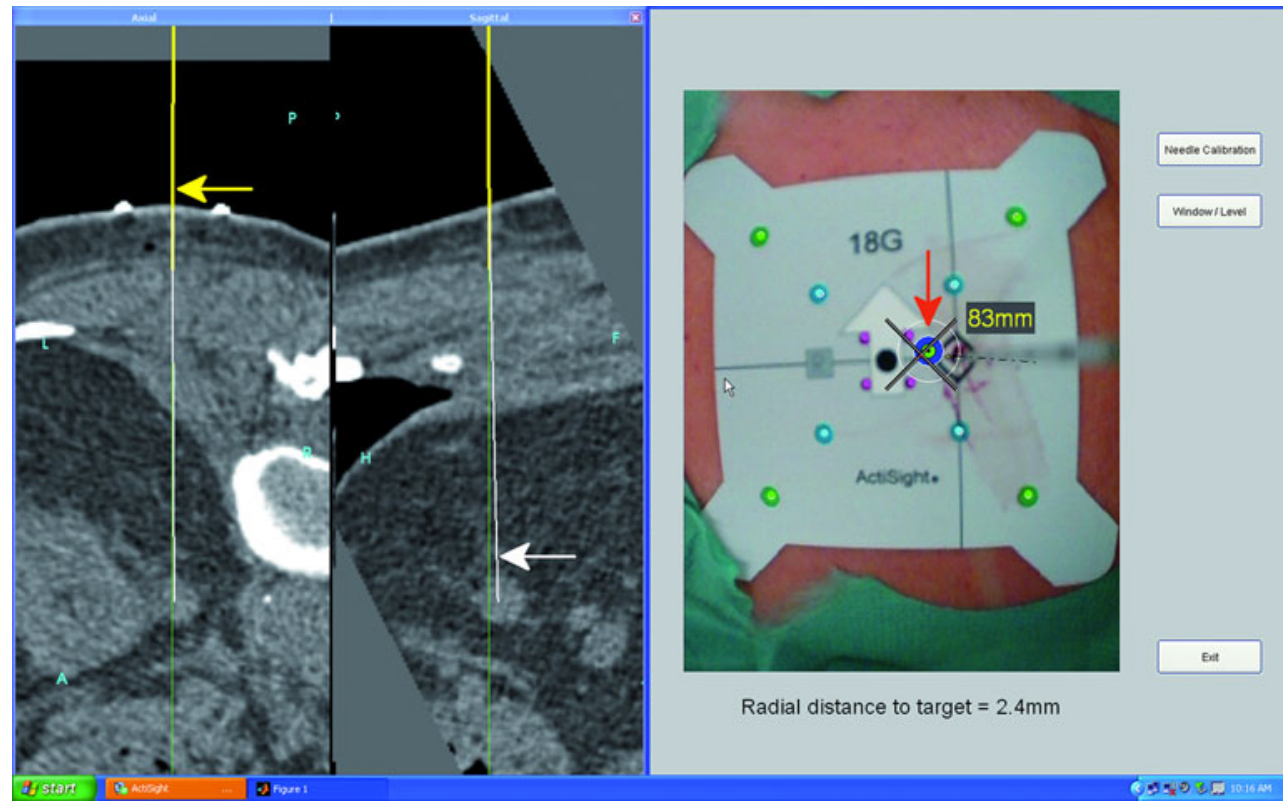

Fig. 2 Biopsy of a pararenal mass (tip of white line on the left side of the screen). On the screenshot of the navigation system, the yellow line depicts the needle and its actual position (yellow arrow), and the white line shows the planned needle path (white arrow). Targeting is possible via following the white line on the biplanar orthogonal view on the left side or via centering the green dot (red arrow) on the crossed lines on the right segment of the screen 


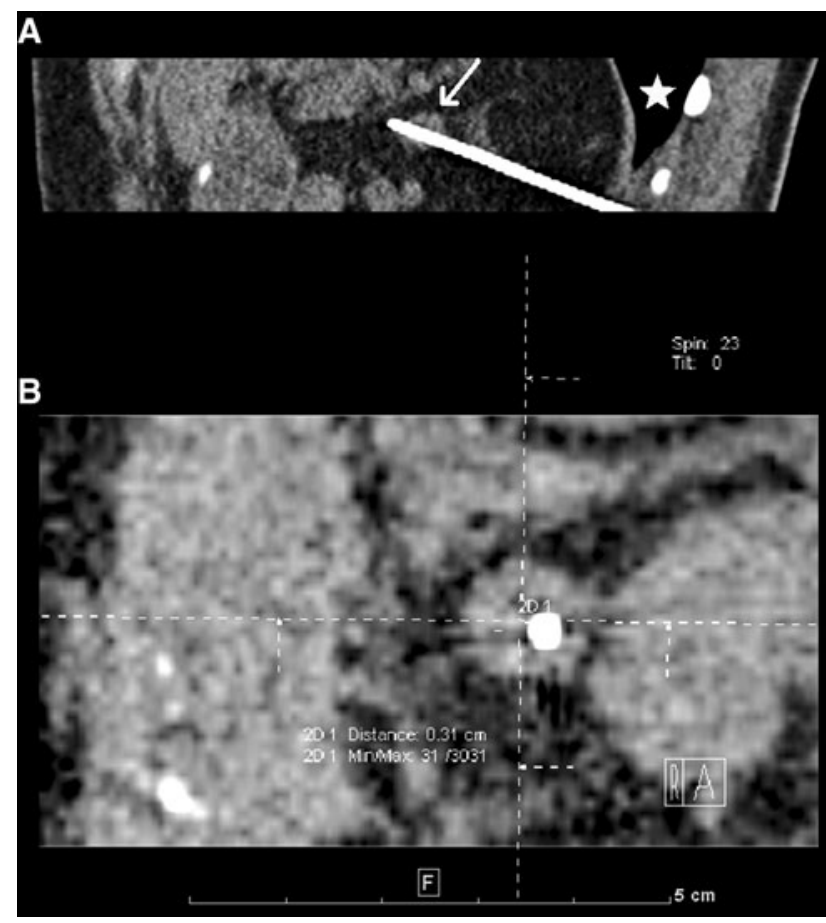

Fig. 3 A Final needle placement of the procedure depicted in Fig. 2 in the pararenal mass (white arrow). An out-of plane approach was chosen to avoid the puncture of the posterior pleural recessus (*). B Orthogonal view to the needle to measure the deviation from the planned needle path

trajectory to the planned needle path. More than one needle passage was defined as pulling the needle back and advancing it again.

\section{Control Group}

In order to rank the intervention time and the number of intermittent CT scans, we randomly chose 15 CT-guided interventions out of the same period as the study group. This cohort comprised seven biopsies, six drainages of fluid collections, one diagnostic aspiration, and one lumbar periradicular infiltration. The mean intervention time was 46.8 min. The mean number of intermittent CT scans during intervention was 4.7 .

\section{Results}

In total, 14 of 16 procedures $(87.5 \%)$ were performed successfully. Ten of 11 biopsies were diagnostic (91\%); 1 was diagnostically unsuccessful due to excessive, painrelated patient movement. In one biopsy, the application of the navigation system had to be aborted due to hypermotility of the subcutaneous tissue, due to which the reference pad could not provide exact determination of the actual position of the probe in the 3D-dataset. This procedure could be finished without use of the navigation system but was counted as a failure of the system. Six biopsies revealed malignancy, and one showed reactive inflammatory tissue. In the latter case, CT controls did not show recurrence 10 months after biopsy. One abscess was evacuated, and the thoracic vertebroplasty was successfully performed via the planned trajectory. Bilateral nephrostomies were performed successfully in an anesthetized patient. In this case, the navigation system was applied because the pyelon was not dilated. The procedure was performed due to severe incontinentia.

Mean deviation of the final probe trajectory to the planned needle path was $1.18 \pm 0.91$ degrees (standard deviation). Mean distance from the probe end to the planned target was $1.98 \pm 1.46 \mathrm{~mm}$ (range 0.5-5.7). Mean number of needle passages was $1.3 \pm 0.76$ (range 1-5). Intervention lasted from patient positioning to postinterventional CT control $1: 15 \mathrm{~h}$ in average (0:35-2:30). No periprocedural complications occurred, and no postprocedural complications were mentioned in the patient records.

\section{Statistical Analysis}

Deviation angle of the probe to the previously planned path and distance from the probe end to the planned target showed a significant correlation $(p=0.01)$. After evaluating both parameters for Gaussian distribution, a multivariate linear model with difference of planned to final trajectory angle and distance from planned target as dependent variables and respiratory movement, in-/outof-plane approach, BMI, intervention time, lesion size, number of needle passages, and needle path length as independent factors was applied.

Intervention time (0.007), respiratory movement $(p=0.008)$, BMI (0.02), and a combination of out of plane approach and respiratory movement $(p=0.003)$ significantly correlated with the deviation angle of the probe trajectory. The $p$ value of out-of-plane approach $(p=0.06)$ was marginally above the range of statistical significance $(p<0.05)$. However, none of the parameters showed a significant correlation with the distance of probe end to planned target (Table 2).

The study group and the control group were compared with respect to intervention time and intermittent CT scans. An unpaired Student's $t$ test revealed a statistically significant difference regarding intervention time in favor of the control group (72.4 vs. $46.8 \mathrm{~min}, p<0.0001)$. The comparison of the number of intermittent CT scans (3.8 in the study group vs. 4.7 in the control group) revealed a $p$ value of 0.07 . However, this was not considered significant, because we set the level of statistical significance at $p<0.05$. 
Table 2 Statistical analysis

\begin{tabular}{|c|c|c|c|c|c|c|c|}
\hline & $\begin{array}{l}\text { Respiratory } \\
\text { Movement }\end{array}$ & $\begin{array}{l}\text { Out-of-plane } \\
\text { approach }\end{array}$ & BMI & $\begin{array}{l}\text { Intervention } \\
\text { time }\end{array}$ & $\begin{array}{l}\text { Lesion } \\
\text { volume }\end{array}$ & $\begin{array}{l}\text { No. of needle } \\
\text { passages }\end{array}$ & $\begin{array}{l}\text { Needle path } \\
\text { length }\end{array}$ \\
\hline $\begin{array}{l}\text { Deviation angle probe to planned } \\
\text { needle path }\end{array}$ & 0.008 & 0.06 & 0.02 & 0.007 & 0.94 & 0.72 & 0.51 \\
\hline $\begin{array}{l}\text { Distance from probe end to } \\
\text { planned target }\end{array}$ & 0.38 & 0.25 & 0.09 & 0.63 & 0.5 & 0.33 & 0.18 \\
\hline
\end{tabular}

$p$ values of the multivariate linear model with deviation angle of probe to planned needle path and distance from probe end to planned target as dependent variables and respiratory movement, in-/out-of-plane approach, BMI, intervention time, lesion size, number of needle passages and needle path length as independent factors. A $p$ value $<0.05$ was considered indicative of a statistically significant correlation

Fig. 4 Repeat biopsy of a large, left iliac mass (M). A solid, contrast-enhancing part (*) of the partially necrotic mass was biopsied via an out-of-plane approach through the greater sciatic foramen

(lymphangioleiomyosarcoma)

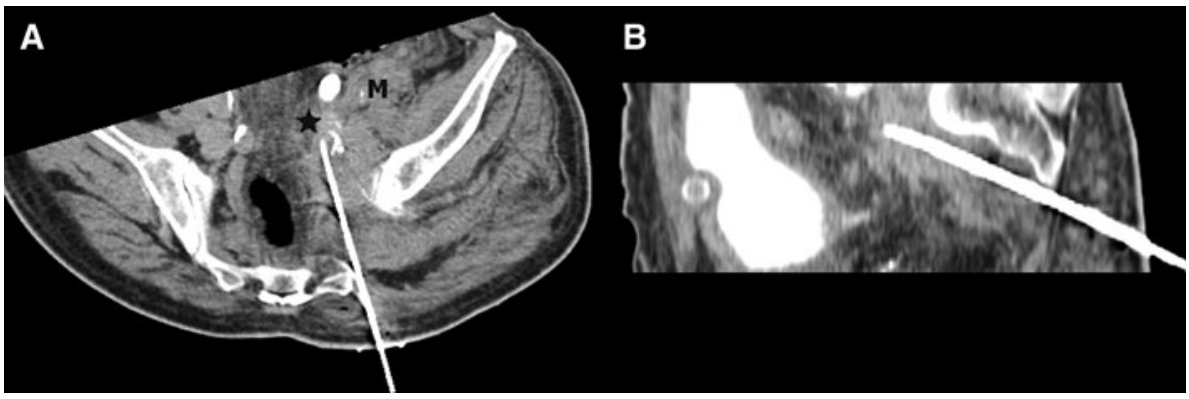

\section{Discussion}

The intention of a navigation system for CT-guided interventions is to avoid deviation of the needle to the planned trajectory providing maximal targeting precision. Likewise, the risk of injuring sensitive structures along the needle path can be minimized. The results of the present study indicate that the application of a CT-based, freehand navigation system for complex percutaneous procedures ensures a high accuracy both in terms of needle path deviation and targeting precision. Targeting precision was excellent from a qualitative and quantitative point of view. The geometrical targeting accuracy of $1.98 \mathrm{~mm}$ in our study is in accordance to the precision of navigated liver punctures reported by Schullian et al. (3.64 mm) [10] and phantom model studies of navigation systems (Stoffner et al.: navigated: $1.94 \mathrm{~mm}$, robotically assisted: $1.69 \mathrm{~mm}$; Appelbaum et al. $2.6 \mathrm{~mm}$ ) [17, 18]. In the mentioned studies, navigation systems were equipped with an aiming device fixing the needle along the planned trajectory. In contrast to these studies, we applied a freehand navigation system without aiming device or robotic assistance. Compared with aiming devices, freehand navigation systems can be used with every standard CT scanner. No additional patient preparation compared with nonnavigated procedures is required. This makes freehand navigated interventions less time-consuming than those with aiming devices or robotic assistance. A further advantage of the applied navigation system is the comparably low price of $\$ 75,000$ USD, which includes disposables for a year. Subsequent disposables amount to $\$ 300$ per procedure. In this context, it must be mentioned that the applied navigation system is only applicable for CT-guided punctures. In contrast, navigation systems of the above-mentioned studies are multipurpose intraoperative navigation systems that run with every crosssectional imaging modality $[10,17,18]$.

In the present study, none of the evaluated parameters showed a statistically significant correlation with the puncture precision (distance from needle tip to planned target). Indeed, several factors were significantly correlated with the angular deviation of the probe trajectory. Two parameters were directly related to the procedure type and region: out-of-plane approach and respiratory motion. Outof-plane approaches are more cumbersome than in-plane approaches, because they require orientation in all three spatial directions (Figs. 3 and 4). Furthermore, 3D reconstructions of the CT data are mandatory in these approaches, and therefore, many operators choose in-plane access whenever possible. Access within the CT plane reduces the degrees of freedom by one to the $\mathrm{x}$ and $\mathrm{y}$ component within the CT plane. In this context, it is conceivable that even navigated, freehand, out-of-plane approaches are associated with a higher needle deviation due to lack of spatial orientation. However, when operators become more familiar with advancing the needle only guided by the visualization on the navigation monitor, needle deviation may further diminish. In this context, we experienced a learning effect both in avoiding pitfalls of the system and in coordinating the needle movements on the two-plane tracking monitor. In contrast, respiratory motion is a challenge both for navigated and conventional image-guided procedures. Internal target motion caused by 
respiratory movement cannot be reliably depicted by surface markers [12]. Apart from invasive internal markers to track respiratory motion [13, 14], recent publications reported promising results with a noninvasive breath-hold control system [19, 20]. In the present study, the majority of procedures in respiratory mobile body parts were successfully performed during breath hold in breathing positions previously practiced with the patient.

In our series, 1 of 14 navigated punctures was unsuccessful. Factors that led to the maximal needle deviation of the series in this case $(5.7 \mathrm{~mm})$ were pain-related patient movement and extensive respiratory motion. In our institution, transcutaneous biopsies are performed routinely under conscious sedation. However, in a small minority of patients, sufficient pain control and sedation may not be achieved with the maximal dosage applied without an attending anesthesiologist [21].

One procedure in a patient with a high BMI (32) had to be aborted due to hypermotility of the subcutaneous tissue. In this case, a reliable navigation could not be ensured in consequence of a highly mobile reference pad on the patient's skin. The puncture could successfully be finalized without navigation. The latter case illustrates a problem of surface fiducials especially in obese patients (BMI > 30). Regarding the applied navigation system, the fiducial markers on the patient's skin are in close proximity to the probe (in fact, the needle runs through the reference pad). Especially in ventral abdominal approaches and obese patients, advancing a coaxial needle through the subcutaneous tissue may cause significant displacement of the patient's skin and the surface fiducials. These effects are reflected in a significant correlation of BMI and angular needle deviation in our series. The effect may be reduced by fixing the skin fiducials with long sterile tape to decrease skin motility.

The mean intervention time for the nonnavigated procedures in a random comparison collective was significantly shorter than in the study group (46.8 vs. $72.4 \mathrm{~min}$ ). However, in this collective, no out-of-plane approaches were required and none of the procedures was performed under general anesthesia. In contrast, 11 of 15 approaches were out of plane in the study group and two procedures were performed under general anesthesia. Furthermore, the optical tracking system was only applied when we expected a technically difficult procedure. Therefore, a certain extension of intervention time compared with standard procedures was expected and tolerated.

The application of a navigation system involves a certain amount of procedures performed with the system to gain and keep experience. In a recently published paper, it could be shown that an experienced user can significantly decrease the time of interventions with an applied navigation system [9]. In the present study, the applied navigation system operated reliably without technical errors, no peri- or postinterventional complications were reported in our series. The mean number of intermittent CT controls was lower in the study group (3.8 vs. 4.7, $p=0.07)$. However, this was not considered significant, because the level of statistical significance was set to $p<0.05$. Diagnostic accuracy of nonnavigated CT-guided biopsies ranges from 64 to $97 \%$ for pulmonary lesions [22, 23]. Sundaram et al. [24] reported an overall accuracy of $95 \%$ for fine-needle aspiration (FNA) of abdominal masses. Accuracy of FNA for pelvic lesions ranges from 80 to $96 \%$ [25, 26]. A series of CT-guided musculoskeletal biopsies depicted an overall accuracy of $71 \%$ [27]. However, mean lesion volume and needle path length is not consistently denoted throughout the mentioned studies.

In conclusion, the results of the present study indicate that the application of an optical tracking system for complex CT-guided procedures provided safe and effective targeting in this series. In our experience, image-guided navigation systems can facilitate CT-guided procedures especially if out-of-plane approaches of small targets with long trajectories or adjacent risk structures are warranted.

Conflict of interest Tilman Schubert, Augustinus L. Jacob, Michele Pansini, David Liu, Andreas Gutzeit, and Sebastian Kos declare that they have no conflict of interest.

\section{References}

1. Gupta S et al (2004) Various approaches for CT-guided percutaneous biopsy of deep pelvic lesions: anatomic and technical considerations. Radiographics 24(1):175-189

2. Long BW (2000) Image-guided percutaneous needle biopsy: an overview. Radiol Technol 71(4):335-359; quiz 360-363

3. Hussain S (1996) Gantry angulation in CT-guided percutaneous adrenal biopsy. Am J Roentgenol 166(3):537-539

4. Odisio BC, Tam AL, Avritscher R, Gupta S, Wallace MJ (2012) CT-guided adrenal biopsy: comparison of ipsilateral decubitus versus prone patient positioning for biopsy approach. Eur Radiol 22(6):1233-1239

5. van Sonnenberg E et al (1981) Triangulation method for percutaneous needle guidance: the angled approach to upper abdominal masses. Am J Roentgenol 137(4):757-761

6. Daly B, Templeton PA (1999) Real-time CT fluoroscopy: evolution of an interventional tool. Radiology 211(2):309-315

7. Prosch H, Stadler A, Schilling M, Bürklin S, Eisenhuber E, Schober E, Mostbeck G (2012) CT fluoroscopy-guided versus multislice CT biopsy mode-guided lung biopsies: accuracy, complications and radiation dose. Eur J Radiol 81(5):1029-1033

8. Mirota DJ, Ishii M, Hager GD (2011) Vision-based navigation in image-guided interventions. Annu Rev Biomed Eng 13:297-319

9. Bale R, Widmann G (2007) Navigated CT-guided interventions. Minim Invasive Ther Allied Technol 16(4):196-204

10. Schullian P et al (2011) Accuracy and diagnostic yield of CTguided stereotactic liver biopsy of primary and secondary liver tumors. Comput Aided Surg 16(4):181-187

11. Rasmus M et al (2007) Robotically assisted CT-based procedures. Minim Invasive Ther Allied Technol 16(4):212-216 
12. Widmann G et al (2010) Respiratory motion control for stereotactic and robotic liver interventions. Int $\mathrm{J}$ Med Robot 6(3):343-349

13. Banovac F et al (2005) Precision targeting of liver lesions using a novel electromagnetic navigation device in physiologic phantom and swine. Med Phys 32(8):2698-2705

14. Maier-Hein L et al (2008) In vivo accuracy assessment of a needle-based navigation system for CT-guided radiofrequency ablation of the liver. Med Phys 35(12):5385-5396

15. Lei $P$ et al (2011) Real-time tracking of liver motion and deformation using a flexible needle. Int J Comput Assist Radiol Surg $6(3): 435-446$

16. Widmann $\mathrm{G}$ et al (2009) Target registration and target positioning errors in computer-assisted neurosurgery: proposal for a standardized reporting of error assessment. Int $\mathrm{J}$ Med Robot 5(4):355-365

17. Stoffner R et al (2009) Accuracy and feasibility of frameless stereotactic and robot-assisted CT-based puncture in interventional radiology: a comparative phantom study. Rofo 181(9):851-858

18. Appelbaum L et al (2011) Electromagnetic navigation system for CT-guided biopsy of small lesions. Am J Roentgenol 196(5):1194-1200

19. Carlson SK et al (2005) CT fluoroscopy-guided biopsy of the lung or upper abdomen with a breath-hold monitoring and feedback system: a prospective randomized controlled clinical trial. Radiology 237(2):701-708

20. Schoth F et al (2010) Evaluation of an interactive breath-hold control system in CT-guided lung biopsy. Rofo 182(6):507-511

21. Higgins TL, Hearn CJ, Maurer WG (1996) Conscious sedation: what an internist needs to know. Cleve Clin J Med 63(6):355-361

22. Gupta S, Madoff DC (2007) Image-guided percutaneous needle biopsy in cancer diagnosis and staging. Tech Vasc Interv Radiol 10(2):88-101

23. Yuan DM et al (2011) Diagnostic efficiency and complication rate of CT-guided lung biopsy: a single-center experience of the procedures conducted over a 10-year period. Chin Med J (Engl) 124(20):3227-3231

24. Sundaram M et al (1982) Utility of CT-guided abdominal aspiration procedures. Am J Roentgenol 139(6):1111-1115

25. Syed R, Bishop JA, Ali SZ (2012) Sacral and presacral lesions: cytopathologic analysis and clinical correlates. Diagn Cytopathol 40(1):7-13

26. Triller J et al (1991) CT-guided biopsy of pelvic masses. Cardiovasc Intervent Radiol 14(1):63-68

27. Hau A et al (2002) Accuracy of CT-guided biopsies in 359 patients with musculoskeletal lesions. Skeletal Radiol 31(6):349-353

28. Schubert $\mathrm{T}$ et al (2012) CT-guided percutaneous biopsy of a mass lesion in the upper presacral space: a sacral transneuroforaminal approach. Cardiovasc Intervent Radiol 35(5):1255-1257 